\title{
Funciones ejecutivas en niños de 5 a 7 años ante el fenómeno bullying 6
}

Diego Alejandro Calle Sandoval

Doctor en Psicología en neurociencias cognitivas aplicadas

Corporación Universitaria Empresarial Alexander Von Humboldt, Colombia Correo electrónico: diacalle54@hotmail.com;

diegocalle116@cue.edu.co
Recibido: 29/05/2019

Evaluado: 29/07/2019

Aceptado: 01/08/2019

Este artículo corresponde a un estudio empírico analítico sobre Bulling y funciones ejecutivas en los primeros años de escolaridad. La medición de las funciones ejecutivas se realizó mediante la prueba Hearts and Flowers en niños de preescolar, y la Batería Neuropsicológica de Lóbulos Frontales y Funciones Ejecutivas (BANFE 2) para los de 6 y 7 años. Además, para medir matoneo se utilizó el Cuestionario sobre bullying (Paredes et al., 2008). La $n(=280)$ reveló un desempeño ejecutivo inferior en los menores con antecedentes de bullying, en comparación con sus pares sin dicha condición. Adicionalmente, aquellos que reportaron ser tanto víctimas como agresores, evidenciaron rendimientos ejecutivos bajos, especialmente en funciones como el control inhibitorio y la empatía. Igualmente, se identificó cierta relación entre la función ejecutiva toma de decisiones y la frecuencia en los actos de agresión en los menores con dicho antecedente.

Palabras clave

Neuropsicología infantil, funciones ejecutivas, bullying, primera infancia.

6 Para citar este artículo: Calle, D.A. (2021). Funciones ejecutivas en niños de 4 a 7 años ante el fenómeno bullying. Informes Psicológicos, 21(1), 87-99 http://dx.doi.org/10.18566/infpsic.v21n1a06 


\section{Executive functions in children from 4 to 7 years old before the bullying phenomenon}

\section{Abstract}

This article corresponds to an analytical empirical study on bullying and executive functions in the first years of schooling. The measurement of executive functions was performed using the Hearts and Flowers test in preschool children and the Neuropsychological Battery of Frontal Lobes and Executive Functions (BANFE 2) for 6 and 7-year-olds. In addition, to measure bullying, the Bullying Questionnaire (Paredes et al., 2008) was used. The $n(=280)$ revealed a lower executive performance in minors with a history of bullying, compared to their peers without said condition. Additionally, those who reported being both victims and aggressors showed low executive performance, especially in functions such as inhibitory control and empathy. Likewise, a certain relationship was identified between the executive function decision-making and the frequency of acts of aggression in minors with said background.

\section{Keywords}

Child neuropsychology, executive functions, bullying, early childhood.

\section{Funções executivas em crianças de 4 a 7 anos perante o fenômeno do bullying}

\section{Resumo}

Este artigo corresponde a um estudo empírico analítico sobre o Bullying e as funções executivas nos primeiros anos de escolaridade. A mensuração das funções executivas foi realizada por meio do teste de Hearts and Flowers em pré-escolares e da Bateria Neuropsicológica de Lobos Frontais e Funções Executivas (BANFE 2) para crianças de 6 e 7 anos. Além disso, para medir 0 bullying, foi utilizado 0 Bullying Questionnaire (Paredes et al., 2008). $0 \mathrm{n}(=280$ ) revelou um desempenho executivo inferior em menores com histórico de bullying, em comparação com seus pares sem essa condição. Além disso, aqueles que reportaram ser vítimas e agressores apresentaram baixo desempenho executivo, principalmente em funções como controle inibitório e empatia. Da mesma forma, foi identificada certa relação entre a função decisória executiva e a frequência de atos de agressão em menores com 0 referido antecedente.

\section{Palavras chave}

Neuropsicologia infantil, funções executivas, bullying, primeira infância. 


\section{ntroducción}

La violencia incide en el desarrollo de las conexiones del sistema límbico y la corteza pre frontal al aumentar los niveles de cortisol que inhiben la acción dopaminérgica del desarrollo favoreciendo conductas de agresión en edades posteriores (Waller, Hyde, BaskinSommers et al., 2017). Igualmente, este fenómeno suele derivar en patologías de la conducta y funciones ejecutivas como la cognición social, el control inhibitorio, flexibilidad cognitiva y memoria de trabajo (Monks, Smith \& Swettenham, 2005). Además, dicho hipodesarrollo ejecutivo se relaciona con alteraciones en la sustancia blanca que vincula el sistema límbico con la región orbitofrontal y el giro cíngulo sobre todo en situaciones de violencia como el Bullying (Holmes, Kim-Spoon \& Deater-Deckard, 2016).

Nathaniel, Riggs, Jahromi, Razza \& Dilworth-Bart (2007) describen los efectos del abandono, el maltrato y otras formas de violencia en el desarrollo neuropsicológico ejecutivo durante la primera infancia como agresión en entornos sociales y escuela. Algo similar encontró Calle (2018) al medir el desarrollo del control inhibitorio en menores de 4 y 5 años víctimas de alguna forma de violencia; hasta un 30\% de disminución en el rendimiento ejecutivo con respecto al grupo control.

Musso (2010) comparó en Argentina el desarrollo de funciones ejecutivas en niños bajo condiciones de riesgo como violencia y altos niveles de pobreza. Valoró el desempeño inhibitorio y ejecutivo en general en 80 menores entre 6 y 10 años a través del paradigma simón dice, en el que se evaluó los errores y la demora en el tiempo de respuesta (más de 2 segundos). A diferencia del grupo control, el desempeño de los primeros fue más bajo en las tareas en las que debían inhibir y el tiempo de respuesta más lento.

En el caso de Colombia, Contreras (2013) encontró que el 29\% de los estudiantes de quinto grado y el 15\% de grado noveno han sufrido algún tipo de agresión. Un estudio exploratorio realizado con 3.373 estudiantes en la ciudad de Medellín, encontró que el 58.35\% del total de participantes admitió haber agredido alguna vez. De estos, 48.17\% manifestó ejecutar conductas de agresión al menos con una frecuencia de una vez al mes. Por otro lado, el 63.30\% del total afirman haber sido alguna vez víctimas de algún tipo de agresión como intimidación (Bromberg, 2013). En Cali, a 2.542 estudiantes de sexto, séptimo y octavo grados se les administró el Cuestionario sobre bullying (Paredes et al., 2008). Al final, el 43.6\% manifestó haber agredido alguna vez a un compañero, mientras que el $51.4 \%$ de participantes indicó haber sido víctima de agresiones. De esa manera, se calculó el índice de bullying en $24.7 \%$ para agresor frecuente y $24.3 \%$ para víctima frecuente, teniendo como criterio que la victimización ocurriera al menos una vez al mes (Paredes et al., 2008). Los últimos años se ha logrado sensibilizar la existencia de bullying o matoneo en un alto porcentaje de la población colombiana, hecho por el cual los estudios psicométricos se han enfocado en este tema en las ciudades grandes (Velásquez \& Pineda, 2016). 


\section{Desarrollo neuropsicológico de funciones ejecutivas}

Diamond (2006) reconoce tres precursores de las funciones ejecutivas: memoria de trabajo, flexibilidad cognitiva e inhibición. Lo anterior se sustenta entre los 3 y 5 años con un acelerado crecimiento dendrítico en las neuronas piramidales de la lámina III de la región dorsal lateral de la corteza pre frontal. Así mismo, el metabolismo de la glucosa en dicha región alcanza niveles tan altos como el de la corteza de un adulto. Para el segundo año, los infantes comienzan a mantener cierta información que les permita resolver una tarea similar. Dicha tarea de memoria de corto plazo está relacionada estrechamente con la corteza temporal basal. Es decir, que logran mantener por mayor tiempo el autocontrol, interiorizando reglas básicas sobre algunos comportamientos motores simples. Igualmente emerge una cierta capacidad de flexibilidad cognitiva que le facilitará al infante construir una teoría de la mente e interpretación de la intención en el otro. Se constituye así la inhibición en la piedra angular sobre la cual se van edificando las funciones ejecutivas a lo largo del desarrollo (Calle, Jiménez \& Romero, 2018).

En general existen dos formas del proceso madurativo de la corteza pre frontal, los progresivos y regresivos. Los primeros hacen referencia a la proliferación celular, el crecimiento de los conos dendríticos y la mielinización. Los regresivos son la pérdida neuronal o apoptosis que se acentúa en la primera infancia, llevando al cerebro a un desarrollo del $90 \%$ a los cinco años de edad (Calle,
2016). La sustancia gris aumenta desde el nacimiento hasta los doce años en promedio; entre los 5 y 11 años las láminas más gruesas son las parietales y la región dorsolateral pre frontal. Respecto a la sustancia blanca, ésta no posee un patrón uniforme de crecimiento; sin embargo, se evidencia más desarrollo a nivel pre frontal en la zona dorsal que en la orbital. El incremento de la sustancia blanca se relaciona directamente con la mielinización de las vías corticotalámicas de la corteza pre frontal. La mielinización de la región orbital finaliza mucho antes que la dorsolateral, por lo que es evidente que ambas poseen raíces filogenéticas y funcionales distintas (Calle, 2017).

Wright y Diamond (2014) aplicaron la prueba Hearts and Flowers a 96 niños entre 6 y 10 años. La parte congruente de la tarea es la presentación de un corazón ante el cual el examinado debe pulsar el botón del mismo lado. La fase incongruente muestra una flor ante la que se exige al menor pulsar el lado opuesto. Entre los resultados hallaron que la capacidad de inhibir mejora con la edad y la aparición de la memoria de trabajo permite que el rendimiento sea mayor cuando la tarea comienza por la fase incongruente. Por tanto, las funciones inhibitoria y memoria operativa son independientes en su desarrollo durante la etapa pre escolar.

Sin duda, lo anterior evidencia cómo la maduración de la región dorsolateral pre frontal en los primeros años se ve influida por procesos etológicos como el apego, pautas de crianza y niveles sociales de bienestar o violencia (Calle, 2012). Partiendo de estas consideraciones, la presente investigación tuvo como objetivo establecer las diferencias en el 
desempeño ejecutivo en niños de 5 a 7 años víctimas, agresores y no participantes directos del fenómeno de bullying en la ciudad de Armenia.

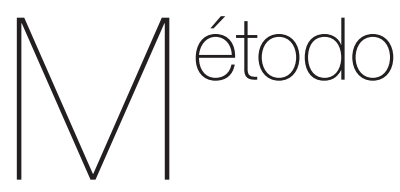

La investigación fue un estudio empírico analítico de alcance descriptivo comparativo, no experimental de corte transversal.

\section{Población}

Participaron 280 niños entre los 5 y los 7 años, vinculados a los centros educativos públicos de la ciudad de Armenia, Quindío. Para la muestra se excluyeron los que tenían antecedentes neurológicos, médicos o retardo madurativo del desarrollo.

\section{Instrumentos}

Se utilizó el Cuestionario sobre bullying (Paredes et al., 2008), que busca identificar menores que pueden clasificarse como agresores dentro del entorno escolar, víctimas y a aquellos que no tienen antecedentes de bullying, a partir de una escala de auto reporte con variables nominales y frecuencias para la ocurrencia de los episodios de violencia (Paredes et al., 2008). Las funciones ejecutivas fueron medidas a través del reactivo Hearts and Flowers para niños de preescolar (5 años y grado cero) desarrollada por Diamond.
El test computarizado presenta un corazón rojo o una flor roja en el lado izquierdo o derecho de la pantalla. Así, dicho test evalúa el control inhibitorio en tres bloques: en el bloque de congruencia, se instruye al niño a presionar la caja de respuesta en el mismo lado del estímulo (corazón) y se otorga un máximo de cuatro puntos; en el bloque de incongruencia, se instruye al niño a presionar la caja de respuesta en el lado opuesto al estímulo (flor) dando un máximo de ocho puntos; finalmente, en el bloque mixto, se instruye al niño a cambiar de forma flexible entre las dos reglas empleadas en los bloques anteriores, de manera que el estímulo puede ser un corazón o una flor, y se otorga un máximo de dieciocho puntos. Para valorar el desempeño ejecutivo en menores de 6 y 7 años se utilizó el BAMFE 2 de Flores, Ostrosky y Lozano (2012). Este consta de quince pruebas que evalúan diferentes procesos asociados con las funciones ejecutivas: frontal orbitomedial, prefrontal anterior y prefrontal dorsolateral.

\section{Procedimiento}

Tras la firma del consentimiento informado, se asignaron jornadas para la aplicación de las dos pruebas psicológicas previamente descritas de forma consecutiva: cuestionario de bullying (alrededor de 16 preguntas relacionadas con las conductas agresivas experimentadas o ejecutadas en el contexto escolar) y una prueba de medición de funciones ejecutivas, de acuerdo con el grupo etario al que pertenecía cada participante. Una vez aplicados los protocolos se procedió al análisis de las variables. Inicialmente, se describieron cada una de éstas, luego se compararon las medidas de dispersión 
entre cada variable de interés, prueba de hipótesis paramétricas y correlaciones.

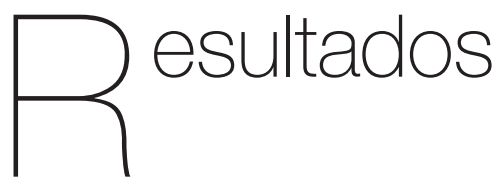

Del total de 280 menores que participaron en el estudio, la mitad (140) eran niñas y la otra mitad niños, 46 tenían 5 años, 103 tenían 6 años y 131 tenían 7 años. 55 De ellos se encontraban cursando preescolar, 134 estaban cursando primer grado y 91 cursaban segundo grado de primaria. Sobre los resultados de la medición del bullying, se encontró que 59 (menos del 25\%) reportaron haber sido víctimas y/o victimarios. De estos, la mayoría (35\%) indicó haber sido agredidos algunas veces, mientras que la minoría 4 aseguró ser agresores de forma frecuente. De aquellos niños que reportaron haber sido víctimas de agresión escolar, más de la mitad manifestaron que su posición de víctimas es ocasional, mientras que los demás se consideran víctimas frecuentes de matoneo.

Respecto al desarrollo neuropsicológico del funcionamiento ejecutivo, de la medición en memoria de trabajo y control inhibitorio en los niños de preescolar, la diferencia por sexo fue muy pequeña en favor de las niñas. Lo anterior era esperado ya que, de acuerdo con Diamond (2006) y Calle (2018), durante los primeros cinco años la memoria de trabajo y el control inhibitorio forjan las bases del funcionamiento ejecutivo de manera paralela a partir del crecimiento de la sustancia blanca entre los 3 y 5 años en condiciones de desarrollo normal; por ello, la media estuvo por encima de 25 y muy cerca de 30 aciertos. Igualmente, la ligera diferencia en favor de las mujeres puede deberse a la rápida maduración cortical de la corteza dorsolateral y orbitofrontal en especial con procesos de cognición social los cuales emergen más rápido en niñas gracias a variables hormonales (BaronCohen, 2012).

Los menores entre 6 y 7 años valorados con el BANFE 2 demostraron que el desempeño de la región orbitomedial, registró una media de 104 y una desviación de 69; en el caso de la porción dorsolateral, se obtuvo un promedio de 92.9 y una desviación de 10; por su parte, para la región pre frontal anterior se observó una media de 100 y una desviación de 9. El rendimiento ejecutivo total en la muestra fue de 94 con una desviación de 10. Si bien la función empatía como la orbitomedial fue la más alta, también es la de mayor dispersión, hecho que podría deberse a las diferencias en los estados emocionales de los menores, ya que, como se verá más adelante, dicha función disminuye en los menores víctimas y/o agresores en el entorno escolar. Otro aspecto a resaltar del desempeño global es que la función más ejecutiva como la dorsolateral tiende a aumentar con la edad, mientras que la emocional u orbital suele ser más alta en niñas (ver Figura 1). Desde luego, la explicación pasaría por la diferencia en el desarrollo de las áreas orbitofrontales femeninas mediadas por la oxitocina que favorece mayor desarrollo de la teoría de la mente (Calle, 2017; Churchland, 2010). 


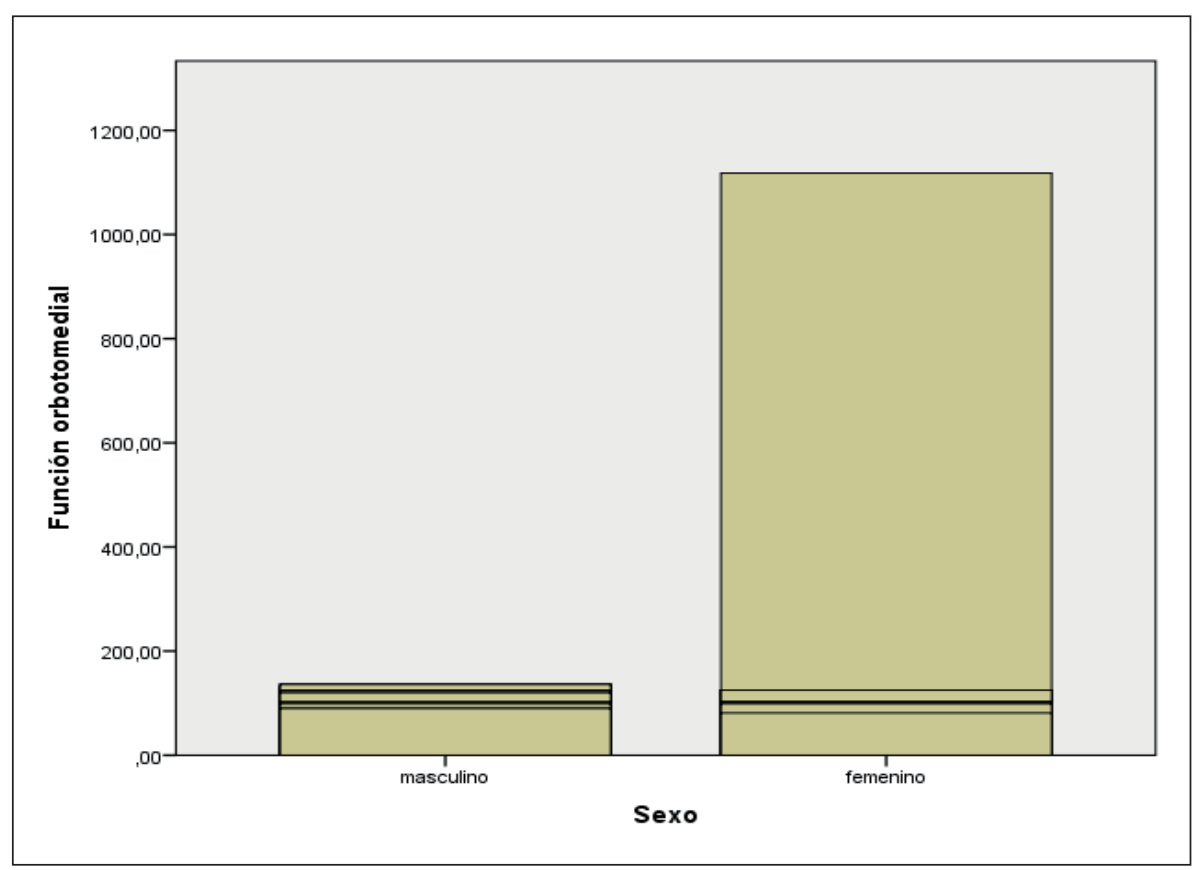

Figura 1. Desempeño ejecutivo promedio de niños y niñas de primero y segundo grado en el dominio orbito-medial de la prueba BANFE 2.

A continuación, los principales ha- inhibitorio frente al reactivo Hearts and llazgos entre las variables bullying y fun- Flowers en menores que se reportan cionamiento ejecutivo en la muestra. como víctima de violencia escolar en disInicialmente, las figuras 2 y 3 muestran tintos niveles de frecuencia. el desempeño ejecutivo en el control

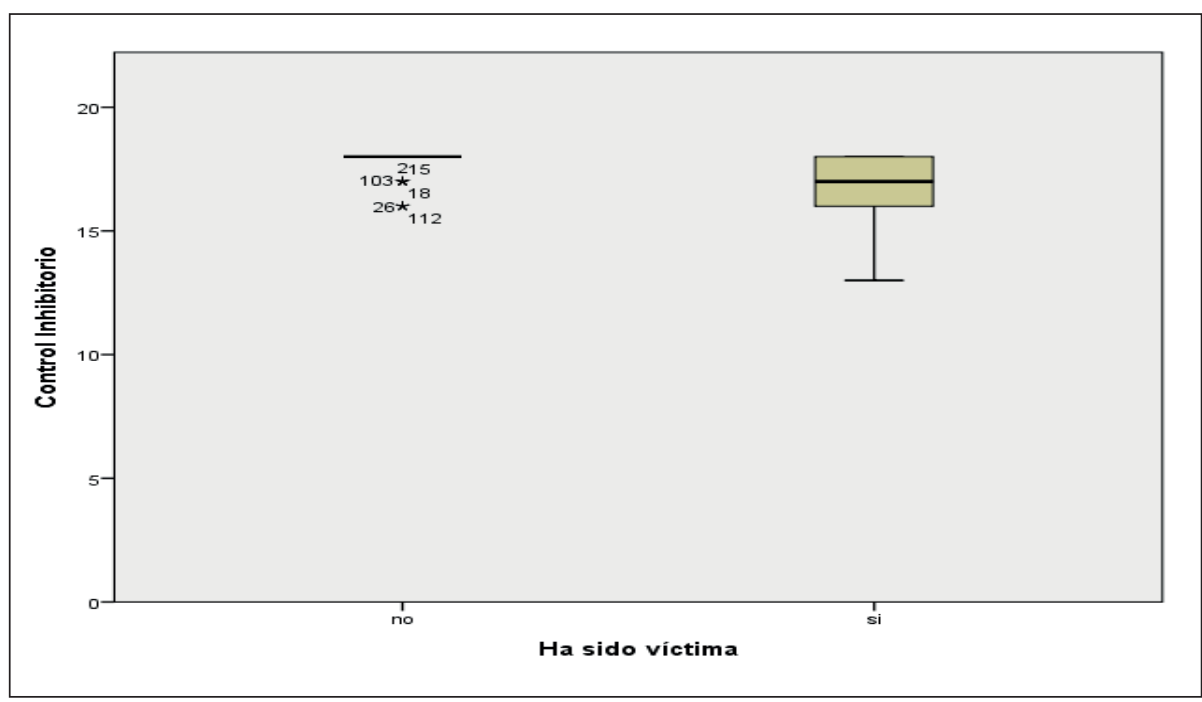

Figura 2. Desempeño ejecutivo promedio de los participantes de grado preescolar en la fase mixta o de control inhibitorio de la prueba Hearts and Flowers, de acuerdo con su condición de víctima de bullying. 


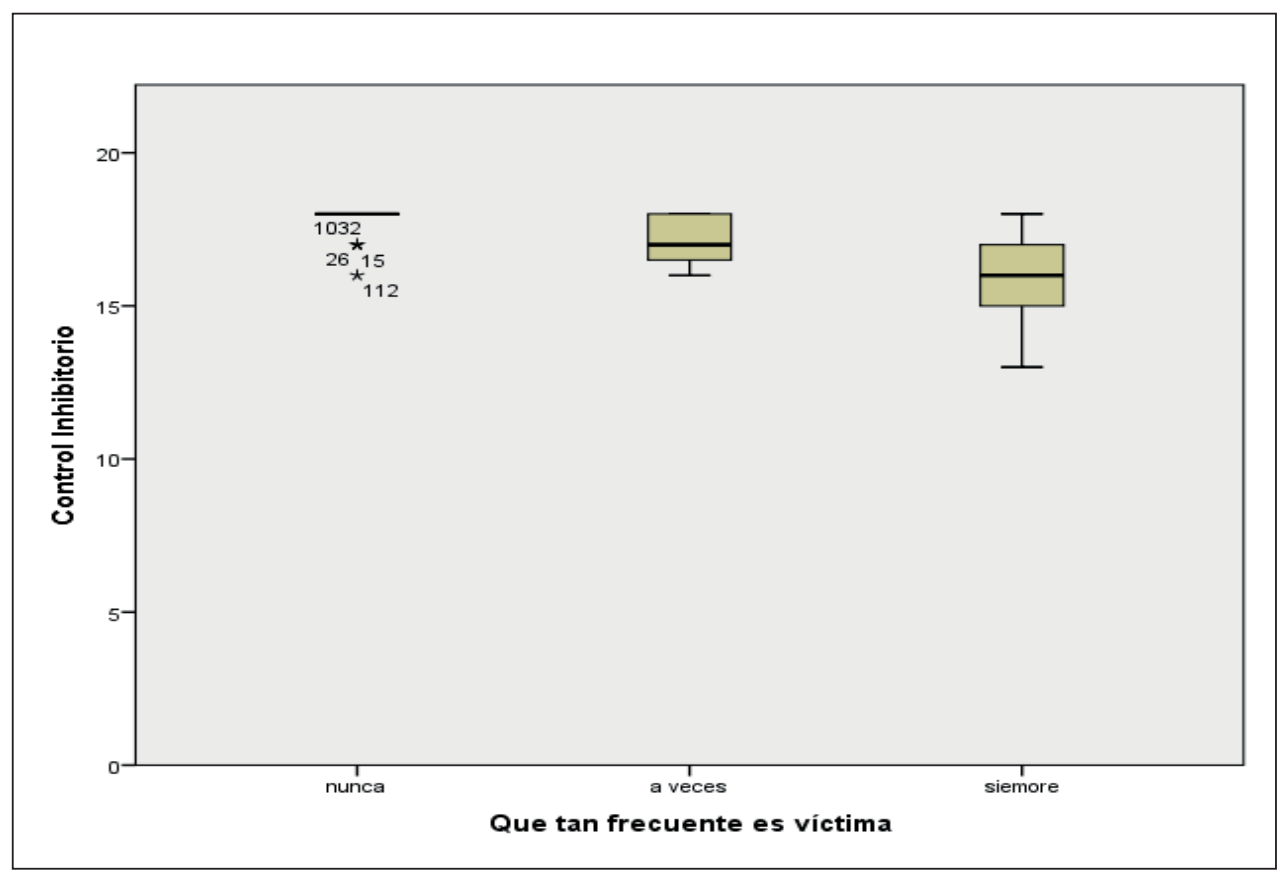

Figura 3. Desempeño ejecutivo promedio de los participantes de grado preescolar en la fase mixta o de control inhibitorio de la prueba Hearts and Flowers, de acuerdo con la frecuencia con la que reportaron ser víctimas de bullying.

En ambas figuras de cajas y bigotes, los sujetos que reportaron nunca ser víctimas de agresión en el colegio indican mejores rendimientos en su media y una distribución más homogénea en los puntajes, ya que las cajas de éstos tienen medias por encima de los otros menores, y sus mínimos y máximos están más cohesionados. Igualmente, de acuerdo con la frecuencia con la que reportaron ser víctimas, se encontró una media inferior y una mayor dispersión en los puntajes de menores que señalaron siempre ser vulnerados, a diferencia de aquellos que informaron ser víctimas de manera ocasional y los que manifestaron nunca recibir matoneo. Es probable que los episodios de estrés impliquen una corteza pre frontal modulada por adrenalina, hecho que puede disminuir la acción inhibitoria de la dopamina en especial en la porción dorsolateral (Cabas, González, \& Mendoza, 2018; Wright \& Diamond, 2014). Resultados similares se observaron con los menores que se reportaron como agresores ocasionales o frecuentes en el entorno escolar. Las Figuras 4 y 5 muestran desempeños más homogéneos quienes no son agresores, en comparación con los agresores. Probablemente esto se asocia con que la estructura del sistema límbico que media entre la corteza pre frontal dorsolateral y la amígdala sea la misma en ambas poblaciones, es decir, el giro cíngulo. Éste, a su vez, es clave en la modulación química del control inhibitorio (Fuster, 2008). 


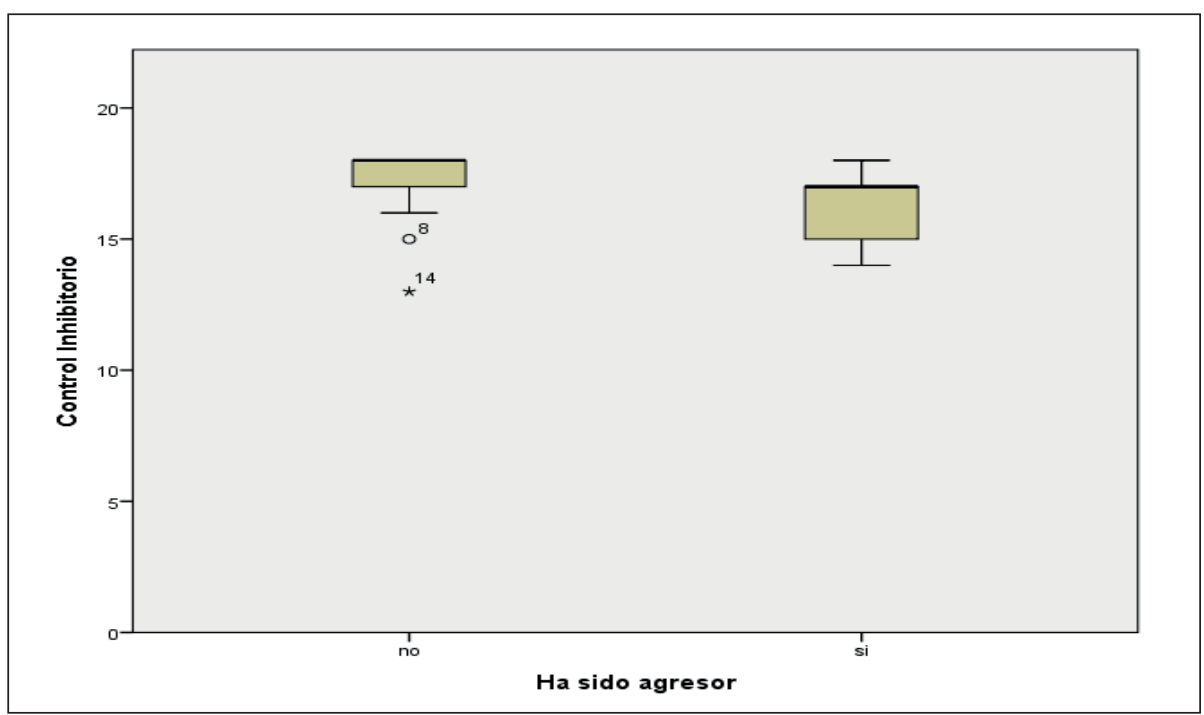

Figura 4. Desempeño ejecutivo promedio de los participantes de grado preescolar en la fase mixta o de control inhibitorio de la prueba Hearts and Flowers, de acuerdo con su condición de agresor.

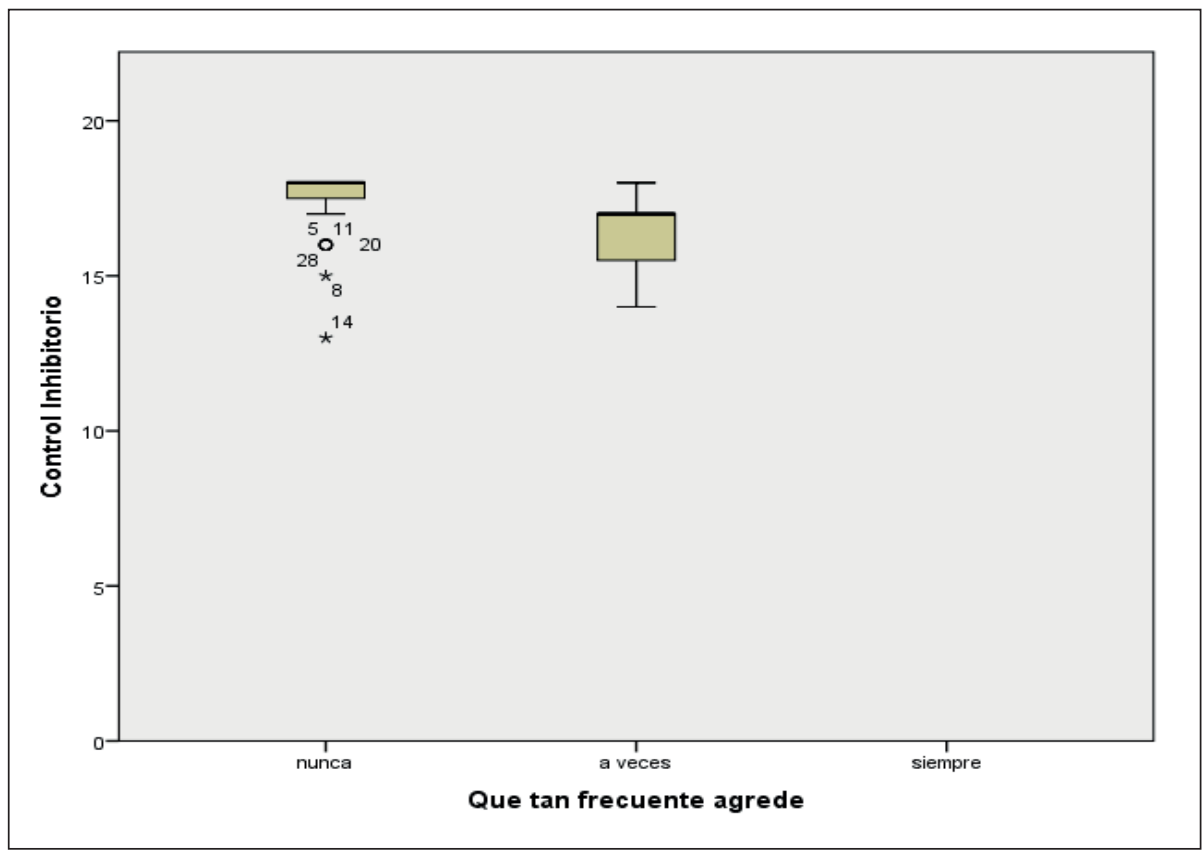

Figura 5. Desempeño ejecutivo promedio de los participantes de grado preescolar en la fase mixta o de control inhibitorio de la prueba Hearts and Flowers, de acuerdo con la frecuencia con la que reportaron ser agresores.

La muestra de infantes entre 6 y 7 años con el BANFE 2 se encontró un rendimiento inferior a medida que se incrementa la frecuencia con la que se agrede (ver Figura 6). Además, el mejor rendimiento se observa en el grupo que no se considera víctima. 


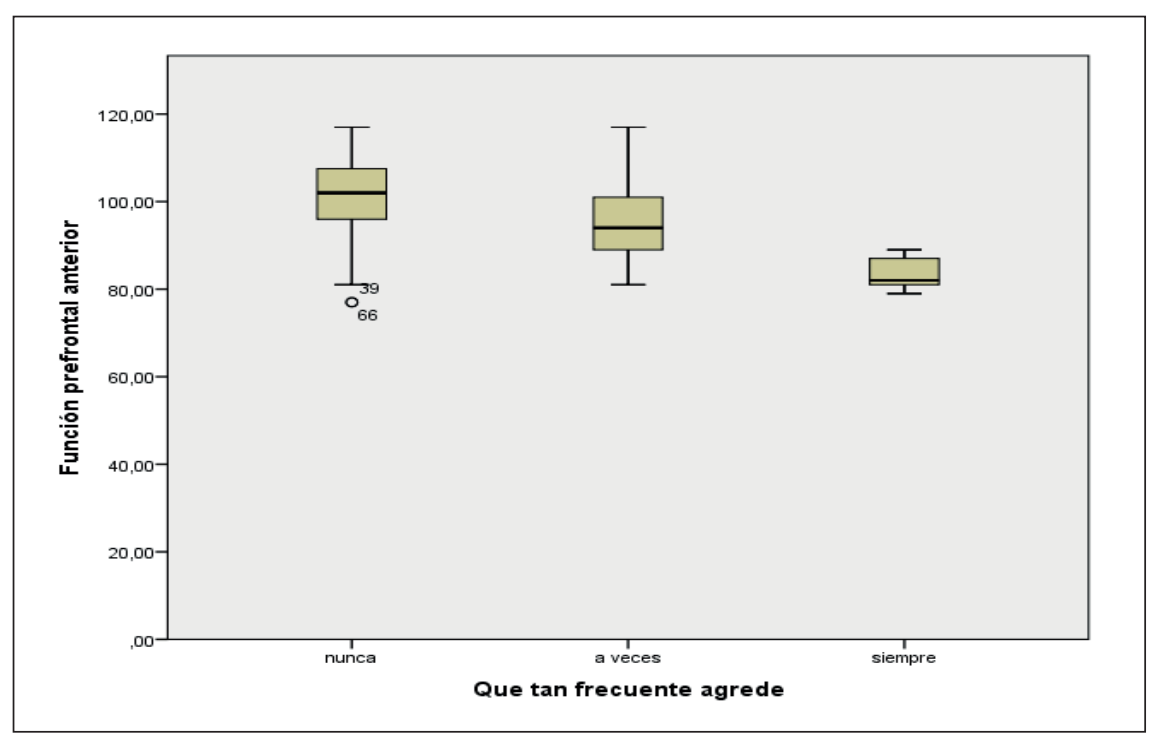

Figura 6. Desempeño ejecutivo promedio de los participantes de primero y segundo grado en el dominio prefrontal anterior de la prueba BANFE 2, de acuerdo con la frecuencia con la que reportaron ser agresores.

La función ejecutiva pre frontal anterior en relación a la frecuencia en que se recibe matoneo indicó desempeños muy semejantes en los niveles de nun$\mathrm{ca}$, siempre y algunas veces (ver Figura
7). Esto significa que ser agresor está acompañado de una toma de decisiones más bajo o menos adaptativo que sus pares víctimas, pasivos o no partícipes.

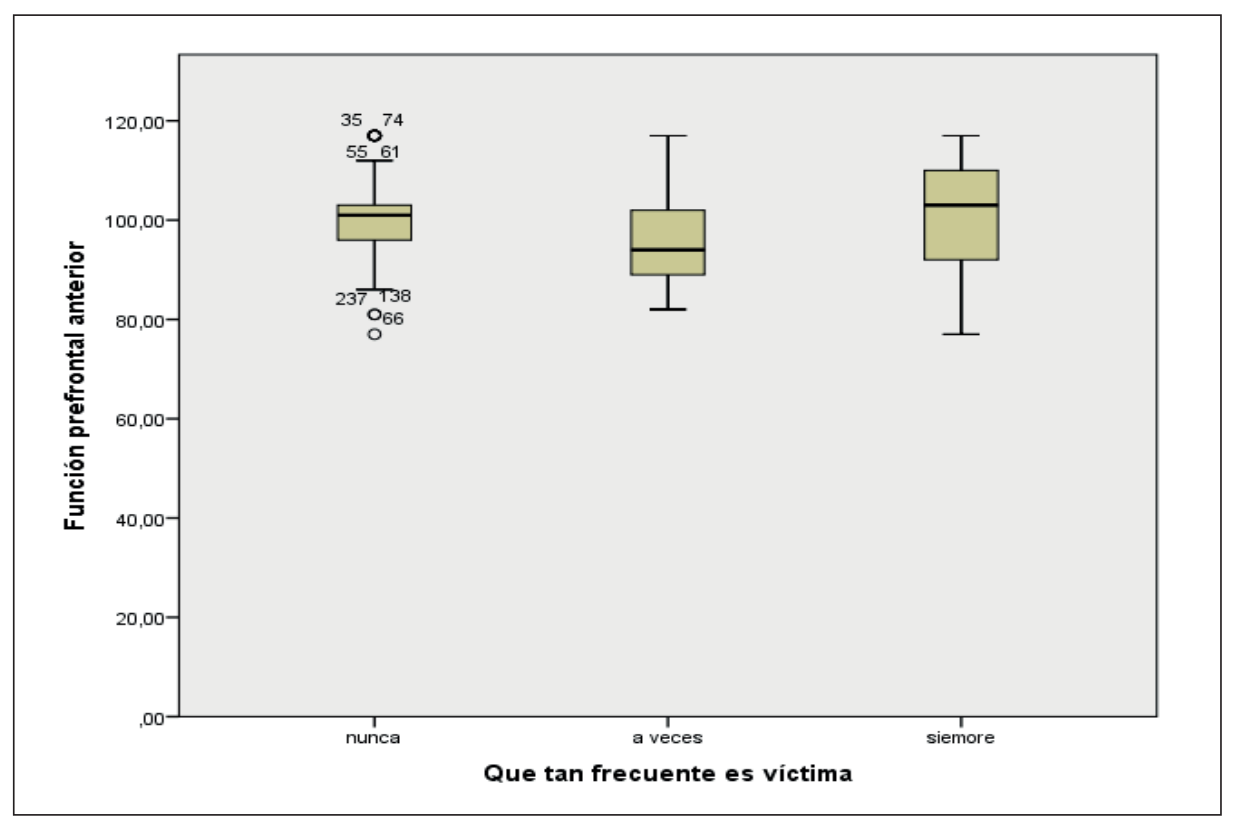

Figura 7. Desempeño ejecutivo promedio de los participantes de primero y segundo grado en el dominio prefrontal anterior de la prueba BANFE 2, de acuerdo con la frecuencia con la que reportaron ser víctimas de bullying. 
Algo semejante se presentó en los resultados de los dominios ejecutivos propios de la estructura dorsolateral, ya que las víctimas y agresores demostraron curvas de desempeño superiores a los agresores. Finalmente, la frecuencia en la agresión y victimización se analiza en relación al funcionamiento ejecutivo total ante la batería BANFE 2. Los resultados del desempeño neuropsicológico muestran el mismo patrón de desempeño observado en los subdominios previamente reportados. Es decir, hay una ejecución más baja en quienes suelen ser siempre agresores o víctimas, en comparación con los ocasionales y los nunca partícipes del matoneo.

Las pruebas de correlación aplicadas permitieron observar una moderada correlación entre la frecuencia de agredir y ser víctima ( $p=.444)$; adicionalmente se halló que dichas variables se correlacionan levemente negativas con el control inhibitorio y el funcionamiento ejecutivo en general ( $p=-$.475). Lo anterior implica que quienes han sido víctimas tienden a ser acosadores o haber ejercido este rol en algún momento. Ahora, el hecho de tener una ligera relación negativa el desempeño ejecutivo con la tendencia a ser víctima o agresor, significa que en efecto un bajo nivel de funcionamiento ejecutivo está asociado con conductas de agresión o matoneo en las edades estudiadas.

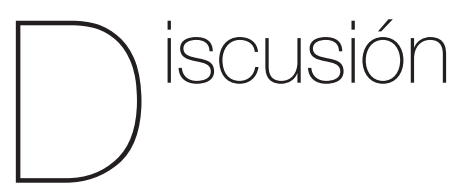

Los resultados indican que cuanto más frecuente se es agresor y/o víctima, es más probable que la capacidad de autocontrol en el menor disminuya. Al disminuir la empatía baja el desempeño ejecutivo global, por ende, también la capacidad de control voluntario del comportamiento y la conducta (Baron-Cohen, 2012; Calle, 2017). Algo similar, pero con niveles poco significativos de correlación, se encuentra entre las mismas variables de bullying (frecuencia de agresión o víctima) y funciones ejecutivas en los niños de 6 y 7 años. Si bien la tendencia es negativa, cuanta más agresión y/o victimización mayor disminución del rendimiento ejecutivo global, no siendo significativos sino leves. Igual que en la literatura revisada, la función ejecutiva más comprometida en sujetos agresores o vulnerados con matoneo suele ser la toma de decisiones.

Para la neuropsicología, es notoria la influencia del control inhibitorio en la capacidad de tomar decisiones, es decir, que el desarrollo de la primera en la etapa preescolar es relevante en la forma como el menor aprende a controlar su comportamiento impulsivo y, por ende, agresivo. Además, es factible sospechar que los menores con falencias inhibitorias también tienen antecedentes de violencia en su vida, ya que la empatía y la teoría de la mente que se desarrollan en la región orbital también suelen aparecer disminuidas.

De otro lado, la investigación permite inferir que:

- La maduración de la sustancia blanca a nivel cortical durante los primeros años se relaciona con la aparición de los precursores de las funciones ejecutivas: el control inhibitorio, la memoria de trabajo y la flexibilidad cognitiva. Igualmente, la empatía que nace de la teoría de la mente propia 
de la región orbitofrontal también participa en el control ejecutivo de la conducta.

- En niñas aparece más rápido la cognición social, hecho que le permite un control inhibitorio más temprano respecto a los varones.

- En menores que han sido víctimas o son agresores el desempeño ejecutivo orbitofrontal o empatía está disminuido.

- Es evidente que el control voluntario de la conducta y el pensamiento en segunda infancia depende en gran medida del desarrollo orbitofrontal o empatía y la maduración de la porción dorsolateral o control inhibitorio.

Finalmente, es un reto para la neuropsicología del desarrollo, la psicología educativa e instituciones educativas realizar programas de promoción del buen trato y prevención de la violencia no sólo en la escuela sino en el hogar a través de las escuelas de padres. Igualmente, deben trabajar en equipo con los centros de salud e IPS o servicios psicológicos que les permita establecer rutas de atención.

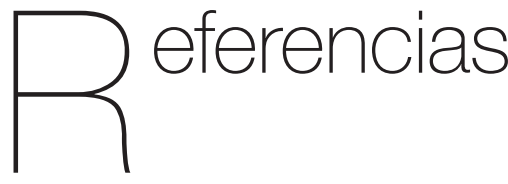

Baron-Cohen, S. (2012). Empatía cero. Buenos Aires, Argentina: Alianza editorial.

Bromberg, P. (2013). Estudios de victimización escolar en Bogotá. En A. Ávila (Ed.), Clima escolar y victimización en Bogotá (2013): encuesta de convivencia escolar (pp. 2548). Bogotá, Colombia: Bogotá Humana.

Cabas, K., González, Y., \& Mendoza, C. (2018). Funcionamiento ejecutivo y depresión en universitarios con normopeso, sobrepeso y obesidad Tipo I. Informes Psicológicos, 18(1), 133-144. http://dx.doi. org/10.18566/ infpsic.v18n1a07

Calle, D. (2012). Apego, Desarrollo y Resiliencia. Informes Psicológicos, 12(1), 25-40. Recuperado de https://dialnet.unirioja.es/ servlet/articulo?codigo $=5229779$

Calle, D. (2016). Desarrollo de funciones ejecutivas y prematuridad: lo que nos cuenta la neuropsicología de la primera infancia. Cuadernos Hispanoamericanos de Psicología, 16(2), 2-22. Recuperado de https://dialnet.unirioja.es/servlet/ articulo?codigo $=5969554$

Calle, D. (2017). Filogenia y desarrollo de funciones ejecutivas. Psicogente, 20(38), 368-381. http://doi.org/10.17081/ psico.20.38.2557

Calle, D. (2018). Funcionamiento ejecutivo en niños de 4 y 5 años víctimas de alguna forma de violencia en el departamento del Quindío. Ocronos Revista Médica y de Enfermería, 3(1), 12-21. Recuperado de https://revistamedica.com/ victimas-violencia-quindio

Calle, D., Jiménez, M., \& Romero, A. (2018). Aproximaciones a la caracterización neuropsicológica de la primera infancia en Colombia. Editorial Universidad Pedagógica y Tecnológica de Colombia UPTC, Tunja 2018. 
Churchland, P. (2010). El Cerebro Moral. Buenos Aires: Paidos.

Contreras, A.P. (2013). El fenómeno de bullying en Colombia. Revista Logos Ciencia \& Tecnología, 4(2), 100-114. Recuperado de http://revistalogos.policia.edu.co/index. php/rlct/article/viewFile/195/206

Diamond, A. (2006). The Early development of executive functions. In E. Bialystok \& F. Craik (Eds.), Lifespan Cognition: Mechanisms of change. (pp. 45-58). New York: Estados Unidos: Oxford University press.

Flores, J., Ostrosky, F. \& Lozano, A. (2012). BANFE: Batería Neuropsicológica de Funciones Ejecutivas y Lóbulos Frontales. México, D.F: Manual Moderno.

Fuster, J. (2008). The Pre frontal Cortex. Burlington USA: Elsevier Academic press.

Holmes, C.J., Kim-Spoon, J. \& Deater-Deckard, K. J. (2016). Linking Executive Function and Peer Problems from Early Childhood Through Middle Adolescence. Abnormal Child Psychology, 44(1), 31-42. https://doi. org/10.1007/s10802-015-0044-5

Monks, C. P., Smith, P. K., \& Swettenham, J. (2005). Psychological correlates of peer victimisation in preschool: social cognitive skills, executive function and attachment profiles. Aggressive Behavior, 31, 571-588. https://doi.org/10.1002/ab.20099

Musso, M. (2010). Funciones ejecutivas: un estudio de los efectos de la pobreza sobre el desempeño ejecutivo. Interdisciplinaria, 27(1), 95-110. Recuperado de http:// www.scielo.org.ar/scielo.php?pid=S1668$70272010000100007 \&$ script $=$ sci_ arttext\&tlng=en
Riggs, N., Jahromi, L., Razza, P., DilworthBart, J. \& Mueller, U. (2007). Executive function and the promotion of socialemotional competence. Journal of Applied Developmental Psychology, 28, 300-309. https://doi.org/10.1016/j. appdev.2006.04.002

Paredes, M., Álvarez, M., Lega, L., \& Vernon, A. (2008). Estudio exploratorio sobre el fenómeno del "Bullying" en la ciudad de Cali, Colombia. Revista Latinoamericana de Ciencias Sociales, Niñez y Juventud, 6(1), 295-317. Recuperado de http:// revistaumanizales.cinde.org.co/rlcsnj/ index.php/Revista-Latinoamericana/ article/view/276/142

Velásquez, J., \& Pineda, L. (2016). Diseño de un instrumento para la identificación del matoneo. Informes Psicológicos, 16(2), 121-141. http://dx.doi.org/10.18566/ infpsicv16n2a08

Waller, R., Hyde, L.W., Baskin-Sommers, A.R. et al. (2017). Interactions between Callous Unemotional Behaviors and Executive Function in Early Childhood Predict later Aggression and Lower Peer-liking in Late-childhood. Journal Abnormal Child Psychology, 45, 597-609. https://doi. org/10.1007/s10802-016-0184-2

Wright, A. \& Diamond, A. (2014). An effect of inhibitory load in children while keeping working memory load constant. Frontiers in Psychology, 5, 1-9. doi: 10.3389/ fpsyg.2014.00213 